\title{
The QUIJOTE-CMB Experiment: studying the polarisation of the Galactic and Cosmological microwave emissions
}

J.A. Rubiño-Martín ${ }^{a, b}$, R. Rebolo ${ }^{a, b, h}$, M. Aguiar ${ }^{a}$, R. Génova-Santos ${ }^{a, b}$, F. Gómez-Reñasco ${ }^{a}$, J.M. Herreros ${ }^{a}$, R.J. Hoyland ${ }^{a}$, C. López-Caraballo ${ }^{a, b}$, A.E. Pelaez Santos ${ }^{a, b}$, V. Sanchez de la Rosa $^{a}$, A. Vega-Moreno ${ }^{a}$, T. Viera-Curbelo ${ }^{a}$, E. Martínez-Gonzalez ${ }^{c}$, R.B. Barreiro ${ }^{c}$,

F.J. $\operatorname{Casas}^{c}$, J.M. Diego ${ }^{c}$, R. Fernández-Cobos ${ }^{c}$, D. Herranz ${ }^{c}$, M. López-Caniego ${ }^{c}$, D. Ortiz ${ }^{c}$,

P. Vielva ${ }^{c}$, E. Artal ${ }^{d}$, B. Aja ${ }^{d}$, J. Cagigas ${ }^{d}$, J.L. Cano ${ }^{d}$, L. de la Fuente ${ }^{d}$, A. Mediavilla ${ }^{d}$,

J.V. Terán ${ }^{d}$, E. Villa $^{d}$, L. Piccirillo ${ }^{e}$, R. Battye ${ }^{e}$, E. Blackhurst $^{e}$, M. Brown ${ }^{e}$, R.D. Davies ${ }^{e}$, R.J. Davis ${ }^{e}$, C. Dickinson ${ }^{e}$, S. Harper ${ }^{e}$, B. Maffei ${ }^{e}$, M. McCulloch ${ }^{e}$, S. Melhuish ${ }^{e}$, G. Pisano ${ }^{e}$,

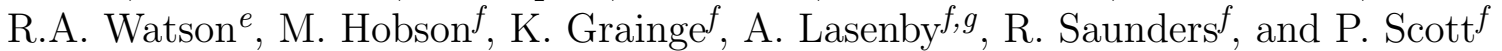

${ }^{a}$ Instituto de Astrofisica de Canarias, C/Via Lactea s/n, E-38200 La Laguna, Tenerife, Spain;

${ }^{b}$ Departamento de Astrofísica, Universidad de La Laguna, E-38206 La Laguna, Tenerife, Spain; ${ }^{c}$ Instituto de Fisica de Cantabria (IFCA), CSIC-Univ. de Cantabria, Avda. los Castros, s/n, E-39005 Santander, Spain;

${ }^{d}$ Departamento de Ingenieria de COMunicaciones (DICOM), Laboratorios de I+D de Telecomunicaciones, Plaza de la Ciencia s/n, E-39005 Santander, Spain;

${ }^{e}$ Jodrell Bank Centre for Astrophysics, School of Physics and Astronomy, University of Manchester, Oxford Road, Manchester M13 9PL, UK;

${ }^{f}$ Astrophysics Group, Cavendish Laboratory, University of Cambridge, Madingley Road, Cambridge CB3 0HE, UK;

${ }^{g}$ Kavli Institute for Cosmology, Univ. of Cambridge, Madingley Road, Cambridge CB3 0HA; ${ }^{h}$ Consejo Superior de Investigaciones Cientificas, Spain

\begin{abstract}
The QUIJOTE (Q-U-I JOint Tenerife) CMB Experiment will operate at the Teide Observatory with the aim of characterizing the polarisation of the $\mathrm{CMB}$ and other processes of Galactic and extragalactic emission in the frequency range of $10-40 \mathrm{GHz}$ and at large and medium angular scales. The first of the two QUIJOTE telescopes and the first multi-frequency $(10-30 \mathrm{GHz})$ instrument are already built and have been tested in the laboratory. QUIJOTE-CMB will be a valuable complement at low frequencies for the Planck mission, and will have the required sensitivity to detect a primordial gravitational-wave component if the tensor-to-scalar ratio is larger than $r=0.05$.
\end{abstract}

Keywords: cosmic microwave background, polarisation, cosmological parameters, early Universe, telescope, instrumentation

\section{INTRODUCTION}

The study of the Cosmic Microwave Background (CMB) anisotropies is one of the most powerful tools in modern cosmology, and it has played a crucial role in our understanding of the Universe. With the latest results from WMAP satellite, ${ }^{1}$ and with the information provided by ground-based experiments such as VSA, ${ }^{2}$ ACBAR, ${ }^{3}$ $\mathrm{CBI},{ }^{4} \mathrm{SPT}^{5}$ or $\mathrm{ACT},{ }^{6}$ it has been possible to determine cosmological parameters with accuracies better than five per cent. ${ }^{7}$ Planck satellite, launched in May 2009, is expected to improve the accuracy on the determination of the cosmological parameters, reaching precisions of less than a percent. ${ }^{8}$

Corresponding author: J.A. Rubiño-Martín. Email: jalberto@iac.es, Telephone: +34922605 276

Ground-based and Airborne Telescopes IV, edited by Larry M. Stepp, Roberto Gilmozzi, Helen J. Hall, Proc. of SPIE Vol. 8444, 84442Y · (c) 2012 SPIE · CCC code: 0277-786/12/\$18 - doi: 10.1117/12.926581 
Until now, the majority of the CMB constraints are obtained from intensity measurements. However, the CMB contains a wealth of information encoded in its polarisation signal. Since the first detection of polarisation by the DASI experiment, ${ }^{9}$ other experiments have provided measurements of the angular power spectrum of the polarisation. ${ }^{10-17}$ Despite their relatively poor signal-to-noise ratio, they still show excellent agreement with the predictions of the standard $\Lambda \mathrm{CDM}$ model.

The standard theory predicts that the CMB is linearly polarized, the physical mechanism responsible for its polarisation being Thomson scattering during the recombination or reionization epochs. Thus, the polarisation state on any direction $\hat{n}$ on sky can be well described by the two Stokes parameters $Q$ and $U$. Full-sky maps of these two parameters can be decomposed into complex spin-2 harmonics

$$
Q(\hat{n}) \pm i U(\hat{n})=\sum_{\ell m} a_{ \pm 2, \ell m} \pm 2 Y_{\ell m}(\hat{n})
$$

However, in practice these coefficients $\left(a_{ \pm 2, \ell m}\right)$ are not used in CMB studies to describe full-sky polarisation maps. Instead, these polarisation maps are decomposed in terms of two scalar components usually called a E-field (gradient) and a B-field (rotational), ${ }^{18,19}$ and which are given by the coefficients

$$
a_{E, \ell m}=-\frac{a_{2, \ell m}+a_{-2, \ell m}}{2}, \quad a_{B, \ell m}=-\frac{a_{2, \ell m}-a_{-2, \ell m}}{2 i}
$$

From here, the angular power spectra can be written as

$$
C_{\ell}^{X Y}=\frac{1}{2 \ell+1} \sum_{m=-\ell}^{m=+\ell} a_{X, \ell m}^{*} a_{Y, \ell m}
$$

where $\mathrm{X}$ and $\mathrm{Y}$ can take the values $\mathrm{T}, \mathrm{E}$, or $\mathrm{B}$. Thus, in addition to the temperature power spectrum $\mathrm{TT}\left(C_{\ell}^{T T}\right)$, we have three parity-independent angular power spectra to describe the polarisation field: the cross-correlation of temperature $\mathrm{T}$ and $\mathrm{E}$ mode, $\mathrm{TE}\left(C_{\ell}^{T E}\right)$; and the auto-correlation of the $\mathrm{E}$ and $\mathrm{B}$ modes, $\mathrm{EE}\left(C_{\ell}^{E E}\right)$ and $\mathrm{BB}$ $\left(C_{\ell}^{B B}\right)$, respectively. All the other combinations (TB and $\mathrm{EB}$ ) are expected to be zero for the CMB field.

The importance of this decomposition is connected with the physics of generation of the CMB anisotropies. If the fluctuations in CMB intensity are seeded by scalar perturbations (i.e fluctuations in the density alone), one would only expect primordial E modes in the CMB polarisation. However, vector and tensor perturbations, like those due to gravitational waves $(\mathrm{GW})$ in the primordial Universe, ${ }^{20}$ are mechanisms that could generate primordial B-modes in the polarisation on large angular scales. Therefore, if we can measure these modes, we may have a unique way to carry out a detailed study of the inflationary epoch. In particular, the energy scale $V$ at which inflation occurred can be expressed in terms of $r$, the ratio of tensor to scalar contributions to the power spectrum, $\mathrm{as}^{21}$

$$
r=0.001\left(\frac{V}{10^{16} \mathrm{GeV}}\right)^{4}
$$

Based on BB upper limits alone, the best current constraint on the inflationary GW background is ${ }^{15} r \leq 0.72$ (95\% C.L.). When combining this information with the measurements of the other three CMB power spectra (TT, TE and EE), the WMAP data ${ }^{16}$ alone gives $r \leq 0.36$ (95\% C.L.). Finally, when BAO and SNIa constraints are included, ${ }^{7}$ we have $r \leq 0.2$ (95\% C.L.). These numbers translate into a constraint of $\lesssim 4 \times 10^{16} \mathrm{GeV}$.

Because of the importance of detecting primordial gravitational waves ${ }^{22,23}$ there is a huge interest to develop experiments to measure (or constrain) the amplitude of B-modes power spectrum of the CMB polarisation. Here we present one of these efforts.

The QUIJOTE (Q-U-I JOint TEnerife) CMB Experiment ${ }^{24}$ is a scientific collaboration between the Instituto de Astrofísica de Canarias, the Instituto de Física de Cantabria, the IDOM company, and the universities of Cantabria, Manchester and Cambridge, with the aim of characterizing the polarisation of the CMB, and other galactic and extragalactic physical processes in the frequency range $10-40 \mathrm{GHz}$ and at angular scales larger than 1 degree. Updated information can be found on the project website. ${ }^{25}$ 


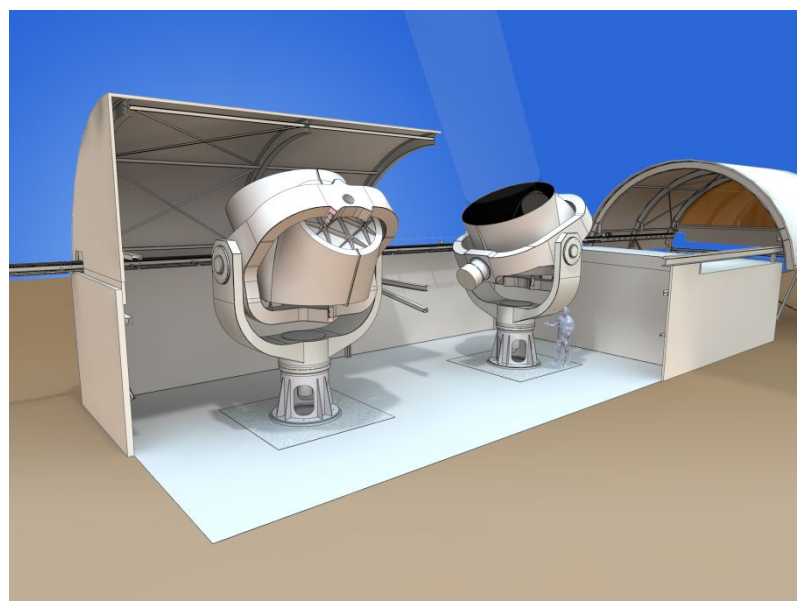

Figure 1. A 3D drawing of the QUIJOTE-CMB experiment dome and the two telescopes.

\section{PROJECT BASELINE}

The QUIJOTE-CMB experiment consists of two telescopes and three instruments (see Fig. 1), which will observe in the frequency range 10-40 GHz with an angular resolution of $\sim 1$ degree, from the Teide Observatory $(2400 \mathrm{~m})$ in Tenerife (Spain). Experience over more than 27 years $^{26}$ with several CMB experiments (Tenerife Experiment, JBO-IAC Interferometer, COSMOSOMAS, Very Small Array) shows that this is an excellent place for CMB observations. The project has two phases already funded:

- Phase I. Construction of the first QUIJOTE-CMB telescope (QT1) and two instruments which can be exchanged in the QT1 focal plane. The first instrument (MFI) is a multichannel instrument providing the frequency coverage between 10 and $20 \mathrm{GHz}$, and it will start commissioning during the summer of 2012 . The second instrument (TGI) will consist of 31 polarimeters working at $30 \mathrm{GHz}$, and it is expected to start operations at the end of 2013 . This phase also includes a two-element interferometer operating at $30 \mathrm{GHz}$, which will be used as a "source-subtractor" facility to monitor and correct the contribution of polarized radio-sources in the final QUIJOTE-CMB maps.

- Phase II. Construction of the second QUIJOTE-CMB telescope (QT2), and a third instrument (FGI) with 40 polarimeters working at $40 \mathrm{GHz}$.

There are also plans for a future Phase III of the project, which considers the construction of a new instrument with at least 100 receivers at W-band. However, this third phase is not funded yet.

Table 1 summarizes the basic (nominal) characteristic of these three instruments in phases I and II. The noise equivalent power (NEP) for one stabilized polarimeter channel is defined here as

$$
\mathrm{NEP}=\sqrt{2} \frac{T_{\mathrm{sys}}}{\sqrt{\Delta \nu N_{\text {chan }}}}
$$

where $T_{\text {sys }}$ stands for the total system temperature, $\Delta \nu$ is the bandwidth and $N_{\text {chan }}$ is the number of channels (computed here as the number of horns times the number of output channels per horn). From here, the noise sensitivity is obtained as $\mathrm{NEP} / \sqrt{t}$, being $t$ the integration time. We note that the system temperature $\left(T_{\text {sys }}\right)$ values appearing in Table 1 have several contributions: the receiver contribution; the estimated contribution of the opto-mechanics; the spillover contribution (i.e., the background contribution when the instrument is placed in the focal plane of the telescope); the atmospheric contribution at the considered frequency; and the CMB contribution $(2.7 \mathrm{~K})$. 
Table 1. Nominal characteristics of the three QUIJOTE-CMB instruments: MFI, TGI and FGI. Sensitivities are referred to Stokes Q and U parameters. See text for details.

\begin{tabular}{|c|c|c|c|c|c|c|c|}
\hline \multirow[b]{2}{*}{ Nominal Frequency [GHz] } & \multicolumn{5}{|c|}{ MFI } & \multirow{2}{*}{$\frac{\text { TGI }}{30}$} & \multirow{2}{*}{$\frac{\text { FGI }}{40}$} \\
\hline & 11 & 13 & 17 & 19 & 30 & & \\
\hline Bandwidth $[\mathrm{GHz}]$ & 2 & 2 & 2 & 2 & 8 & 8 & 10 \\
\hline Number of horns & 2 & 2 & 2 & 2 & 1 & 31 & 40 \\
\hline Channels per horn & 4 & 4 & 4 & 4 & 2 & 4 & 4 \\
\hline Beam FWHM [] & 0.92 & 0.92 & 0.60 & 0.60 & 0.37 & 0.37 & 0.28 \\
\hline$T_{\mathrm{sys}}[\mathrm{K}]$ & 25 & 25 & 25 & 25 & 35 & 35 & 45 \\
\hline $\mathrm{NEP}\left[\mu \mathrm{K} s^{1 / 2}\right]$ & 280 & 280 & 280 & 280 & 390 & 50 & 50 \\
\hline Sensitivity $\left[\mathrm{Jy} \mathrm{s}^{1 / 2}\right]$ & 0.30 & 0.42 & 0.31 & 0.38 & 0.50 & 0.06 & 0.06 \\
\hline
\end{tabular}
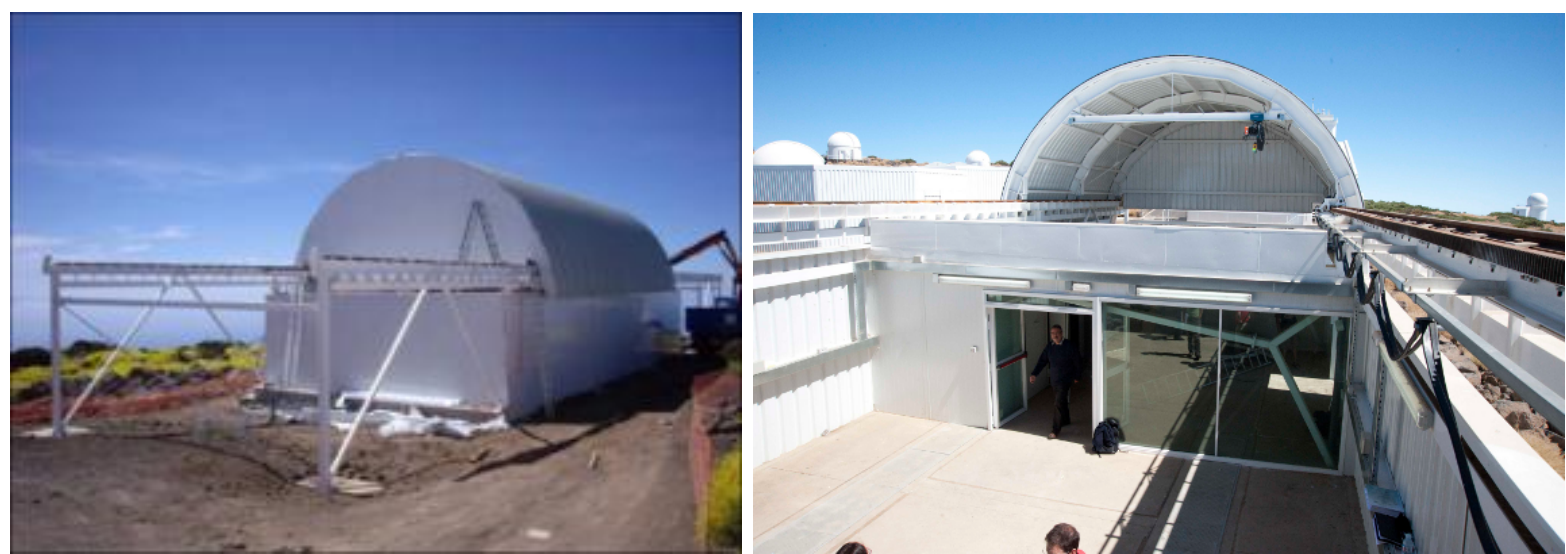

Figure 2. Left: QUIJOTE-CMB enclosure at the Teide Observatory. Right: Inside the QUIJOTE-CMB dome, before the installation of QT1.

\section{EXPERIMENT DESCRIPTION}

\subsection{Telescopes and Enclosure}

The QUIJOTE-CMB experiment consists of two telescopes (hereafter QT1 and QT2) that will be installed inside a single enclosure at the Teide Observatory. The enclosure and the building hosting the control room were finished in June 2009 (see Fig. 2).

The layout of both QT1 and QT2 telescopes is based on an altazimuth mount supporting a primary (parabolic) and a secondary (hyperbolic) mirror disposed in an offset Gregorian Dracon scheme, which provides optimal crosspolarisation properties (designed to be $\leq-35 \mathrm{~dB}$ ) and symmetric beams. Each primary mirror has a $2.25 \mathrm{~m}$ projected aperture, while the secondary has $1.89 \mathrm{~m}$. The system is under-illuminated to minimize side-lobes and ground spillover. Each telescope is mounted on its own platform that can rotate around the vertical axis at a maximum frequency of $6 \mathrm{rpm}$ (i.e., $36 \mathrm{deg} \mathrm{s}^{-1}$ ).

The telescope control software for QT1 was implemented during 2009, and the different observing modes (raster, scanning, tracking, etc.) have been tested. ${ }^{27}$ The construction scheme, as well as the fabrication techniques for QT1 have been already presented. ${ }^{28}$ We note that the QT1 mirrors have been designed to operate up to $90 \mathrm{GHz}$ (i.e., $\mathrm{rms} \leq 20 \mu \mathrm{m}$ and maximum deviation of $d=100 \mu \mathrm{m}$ ). However, QT2 has been specified to have a better surface accuracy, so the telescope could in principle operate up to $200 \mathrm{GHz}$.

The installation of QT1 at the Teide Observatory took place during May 2012 (see Fig. 3).

\subsection{Instruments}

\subsubsection{Multi-frequency Instrument (MFI)}

This is a multi-channel instrument with five independent sky pixels: two operate at 10-14 GHz; the other two at $16-20 \mathrm{GHz}$, and finally a central polarimeter at $30 \mathrm{GHz}$ that is being used as a demonstrator of the second 

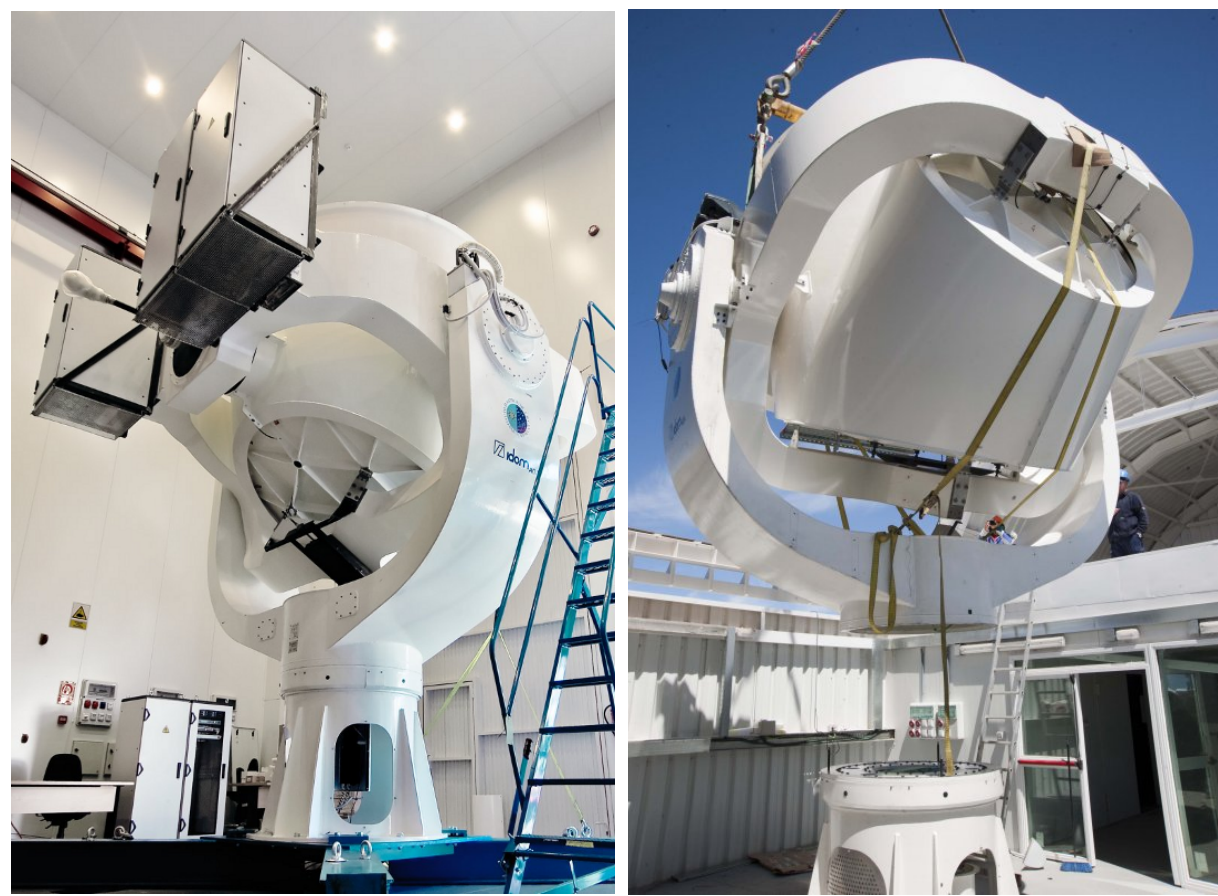

Figure 3. Left: QT1 at the IAC workshop (June 2009). Right: Installation of QT1 at the Teide Observatory (May 3rd, 2012).

instrument during the laboratory tests and commissioning phase. The main science driver for the MFI is the characterization of the Galactic emission. A complete description of the MFI and the details on the software are presented elsewhere. ${ }^{27,29}$ Here, we only provide the basic aspects of the instrument (see Fig. 4).

The optical arrangement includes five conical corrugated feedhorns (designed by the University of Manchester). Each horn feeds a novel cryogenic on-axis rotating polar modulator which can rotate at speeds of up to $1 \mathrm{~Hz}$. We consider two possible operational modes: either continuous rotation of the polarimeters, or discrete changes of the positions of the motors in steps of $22.5^{\circ}$ (note that the polar modulation occurs at four times the rotation angle). The orthogonal linear polar signals are separated through a wide-band cryogenic OrthoMode-Transducer (OMT) before being amplified through two similar LNAs (a Faraday-type module in the case of $30 \mathrm{GHz}$ ). These two orthogonal signals are fed into a room-temperature Back-End module (BEM) where they are further amplified and spectrally filtered before being detected by square-law detectors. All the polarimeters except the $30 \mathrm{GHz}$ receiver have simultaneous "Q" and "U" detection i.e. the 2 orthogonal linear polar signals are also correlated through a $180^{\circ}$ hybrid and passed through two additional detectors. The band passes of these lower frequency polarimeters have also been split into an upper and lower band which gives a total of 8 channels per polarimeter (see Table 1).

The FEM for the low frequency channels was built by the IAC. The receivers for these channels use MMIC $6-20 \mathrm{GHz}$ LNAs (designed by S. Weinreb and built in Caltech). The gain for these amplifiers is approximately $30 \mathrm{~dB}$, and the noise temperature is less than $9 \mathrm{~K}$ across the band. The $30 \mathrm{GHz}$ FEM was built at the University of Manchester, and the design used an existing Faraday module (same as the one used for OCRA-F*). The BEM for the $30 \mathrm{GHz}$ instrument was built by DICOM, with collaboration of IFCA at the simulation level. The cryogenics and the mechanical systems were provided by the IAC.

\subsubsection{Thirty-GHz Instrument (TGI)}

This instrument will be mainly devoted to primordial B-mode science. TGI will be fitted with 31 polarimeters working in the range of $26-36 \mathrm{GHz}$. After the laboratory tests with the $30 \mathrm{GHz}$ polarimeter of the MFI, we found

\footnotetext{
*OCRA-F: http://www.jodrellbank.manchester.ac.uk/research/ocra/ocraf .html.
} 

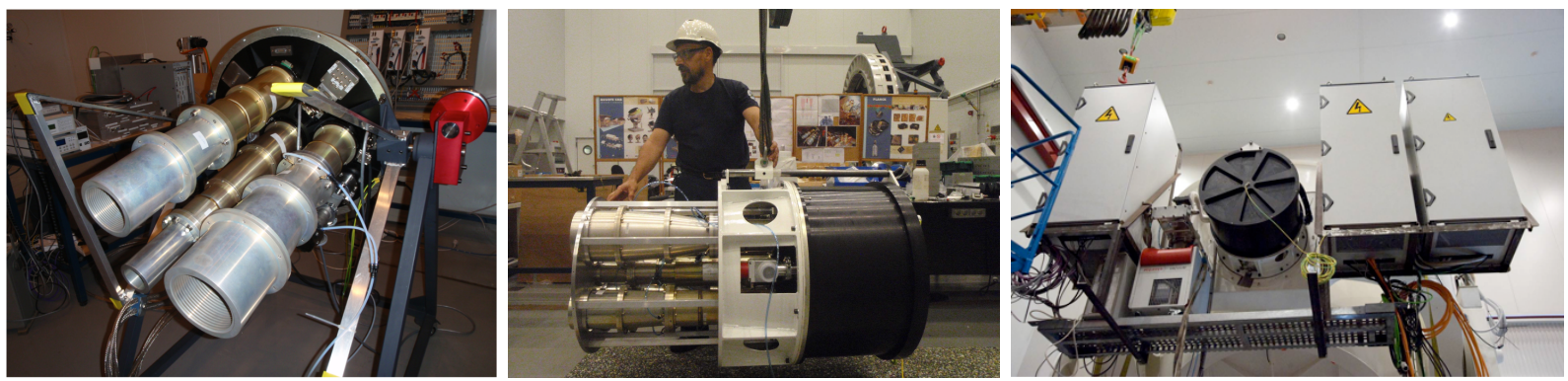

Figure 4. Left: Close view of the MFI, during the integration phase (May 2011). Center: Integration of the MFI in the QT1 focal plane (December 2011). Right: MFI already installed at the QT1 focal plane (January 2012). The electronic boxes controlling the telescope and the instrument are also installed.

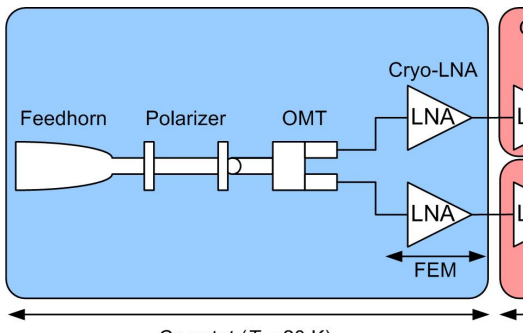

Cryostat $(T=20 \mathrm{~K})$

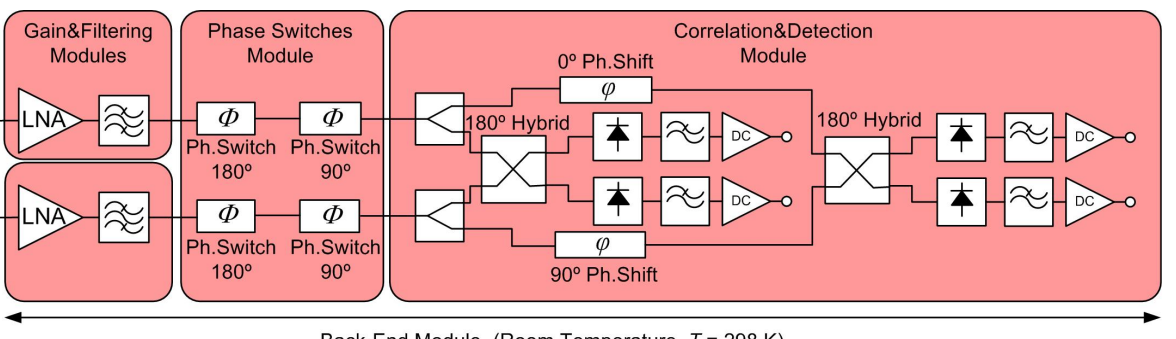

Back-End Module (Room Temperature, $T=298 \mathrm{~K}$ )

Figure 5. Configuration of each of the 31 receivers of the TGI, in the QUIJOTE-CMB experiment.

that the MFI design, based on the spinning polar modulators in a cryogenic environment, is not appropriate for the long-term operations required for the TGI. Thus, we have modified the receiver configuration by replacing the rotating polar modulator with a fixed polarizer. The current new design is presented in Fig. 5. It includes a fixed polarizer and $90^{\circ}$ and $180^{\circ}$ phase switches to generate four polarisation states to minimize the different systematics in the receiver. A detailed description of the system has been already presented. ${ }^{30}$

\subsubsection{Forty-GHz Instrument (FGI)}

Also devoted to primordial B-mode science, the FGI will be fitted with 40 polarimeters working at $40 \mathrm{GHz}$. The conceptual design of a polarimeter chain for the FGI is identical to the one used for the TGI (see Fig. 5).

\subsection{Source subtractor facility}

An upgraded version of the VSA source subtractor (VSA-SS) facility, ${ }^{31}$ which is being carried out by the Cavendish Laboratory and the University of Manchester, will be used to monitor the contribution of polarized radio-sources in the QUIJOTE-CMB maps. The VSA-SS is a two element interferometer, operating at $30 \mathrm{GHz}$, with $3.7 \mathrm{~m}$ dishes and a separation of $9 \mathrm{~m}$ (see Fig. 6). The VSA-SS system only measured one linear polarisation of the incoming radiation, so it is being upgraded to include a half-wave plate (HWP) in front of each of the antennas in order to allow for successive measurements of Stokes Q and U. Here, we use a dielectrically embedded mesh-HWP designed and produced at the University of Manchester (see right panel of Fig. 6).

Using the method described by Tucci et al. $(2004)^{32}$ to simulate the polarisation properties of radio sources at the QUIJOTE-CMB frequencies, we have estimated that in order for the residual source contribution to our measurements be equal to or smaller than the expected B-mode signal for the case of $r=0.1 \mathrm{at} 30 \mathrm{GHz}$, we must remove the effects of all sources whose Stokes I intensity is higher than $300 \mathrm{mJy}$ (see Fig. 7). Our strategy is therefore to measure the $30 \mathrm{GHz}$ Stokes I intensity of known radio sources (e.g., from the GB6 catalogue) and then measure the polarisation of those that we find have Stokes I greater than $300 \mathrm{mJy}$. The total number of sources to be monitored in the whole QUIJOTE-CMB surveyed area will be around 500. The expected polarised flux sensitivity per source of the VSA-SS is $2-3 \mathrm{mJy}$.

An upgrade of this VSA-SS facility to operate at $40 \mathrm{GHz}$ during the Phase II of the project is currently under discussion. 

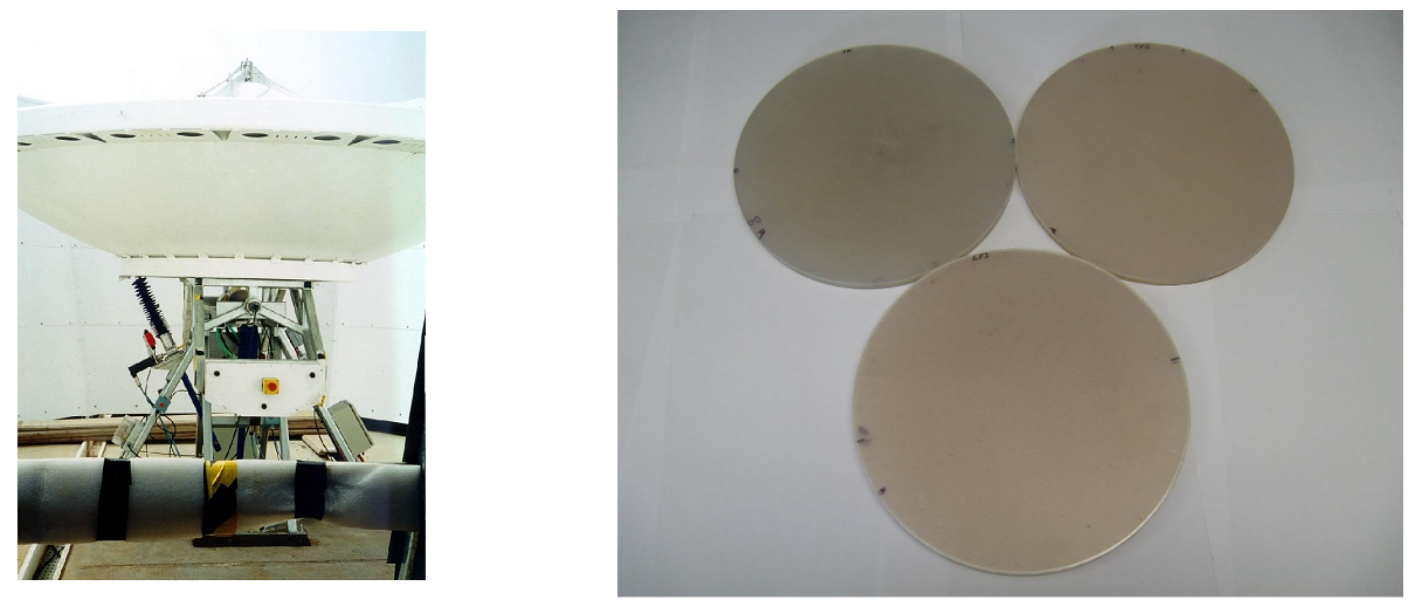

Figure 6. Left: One of the two antennas of the VSA source subtractor. This facility will be re-used to measure the polarisation of radio sources to correct the QUIJOTE-CMB $30 \mathrm{GHz}$ maps. Right: Picture of the HWPs used for the VSA-SS.

\section{SCIENCE GOALS AND SCIENCE CASES}

\subsection{Core science}

The QUIJOTE-CMB experiment has two primary scientific goals:

- to detect the imprint of gravitational B-modes if they have an amplitude $r \geq 0.05$;

- to provide essential information of the polarisation of the synchrotron and the anomalous microwave emissions from our Galaxy at low frequencies $(10-40 \mathrm{GHz})$.

For these scientific objectives, QUIJOTE-CMB will conduct two large surveys in polarisation (i.e., Stokes $\mathrm{Q}$ and U maps):

i) a shallow "Galactic" survey. It will cover around $10000 \mathrm{deg}^{2}$ of sky. It is expected to be finished after $2-3$ months of effective observing time with each instrument, reaching sensitivities of $\sim 10-15 \mu \mathrm{K}$ per one degree beam in the Stokes Q and U maps with the MFI (11-19 GHz), and $\lesssim 3 \mu \mathrm{K}$ per beam with the TGI and FGI at 30 and $40 \mathrm{GHz}$.

ii) a deep "Cosmological" survey. It will cover around $3000 \mathrm{deg}^{2}$. Here, we shall reach sensitivities of $\sim 3-4 \mu \mathrm{K}$ per one degree beam after one year of effective observing time with the MFI (11-19 GHz), and $\lesssim 1 \mu \mathrm{K}$ per beam with TGI and FGI at 30 and $40 \mathrm{GHz}$.

According to these nominal sensitivities, QUIJOTE-CMB will provide one of the most sensitive $11-19 \mathrm{GHz}$ measurements of the polarisation of the synchrotron and anomalous emissions on degree angular scales. This information is extremely important given that B-modes are known to be sub-dominant in amplitude as compared to the Galactic emission, ${ }^{33}$ as illustrated in Fig. 7. The QUIJOTE-CMB maps will also constitute an unique complement of the Planck satellite ${ }^{\dagger}$, helping in the characterization of the Galactic emission. In particular, the combination of Planck and QUIJOTE-CMB will allow us: (a) to determine synchrotron spectral indices with high accuracy, and to fit for curvature of the synchrotron spectrum to constrain CR electron physics; ${ }^{34}$ (b) to study the large-scale properties of the Galactic magnetic field; ${ }^{35}$ or (c) to assess the level of a possible contribution of polarized anomalous microwave emission. ${ }^{36,37}$

Using the MFI maps from the deep survey, we plan to correct the high frequency QUIJOTE-CMB channels (30 and $40 \mathrm{GHz}$ ) to search for primordial B-modes. As an illustration, Fig. 8 presents two cases. The left panel shows the scientific goal for the angular power spectrum of the $\mathrm{E}$ and $\mathrm{B}$ modes after 1-year of effective observing time, assuming a sky coverage of 3000 square degrees, with the TGI only. In this particular case, the final noise

${ }^{\dagger}$ Planck: http://www.rssd.esa.int/index.php?project=Planck 


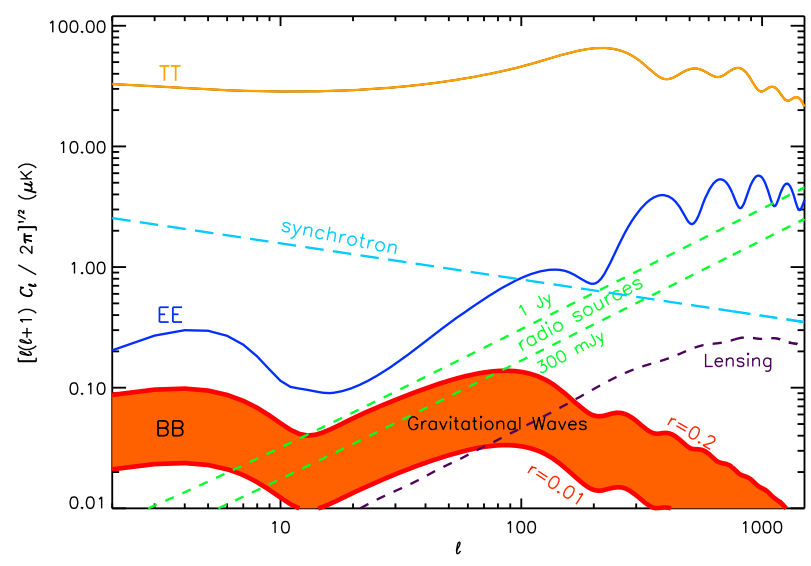

Figure 7. Expected foreground contamination in the $30 \mathrm{GHz}$ QUIJOTE-CMB frequency band. It is shown the contribution of polarized synchrotron emission and radio-sources for the case of subtracting sources down to 1 Jy in total intensity (upper dashed line for radio-sources) and $300 \mathrm{mJy}$ (lower dashed-line). The physical models for these emissions are described in. ${ }^{33}$ The shaded red area shows the expected level of primordial gravitational waves for $r$ in the range $0.01-0.2$.
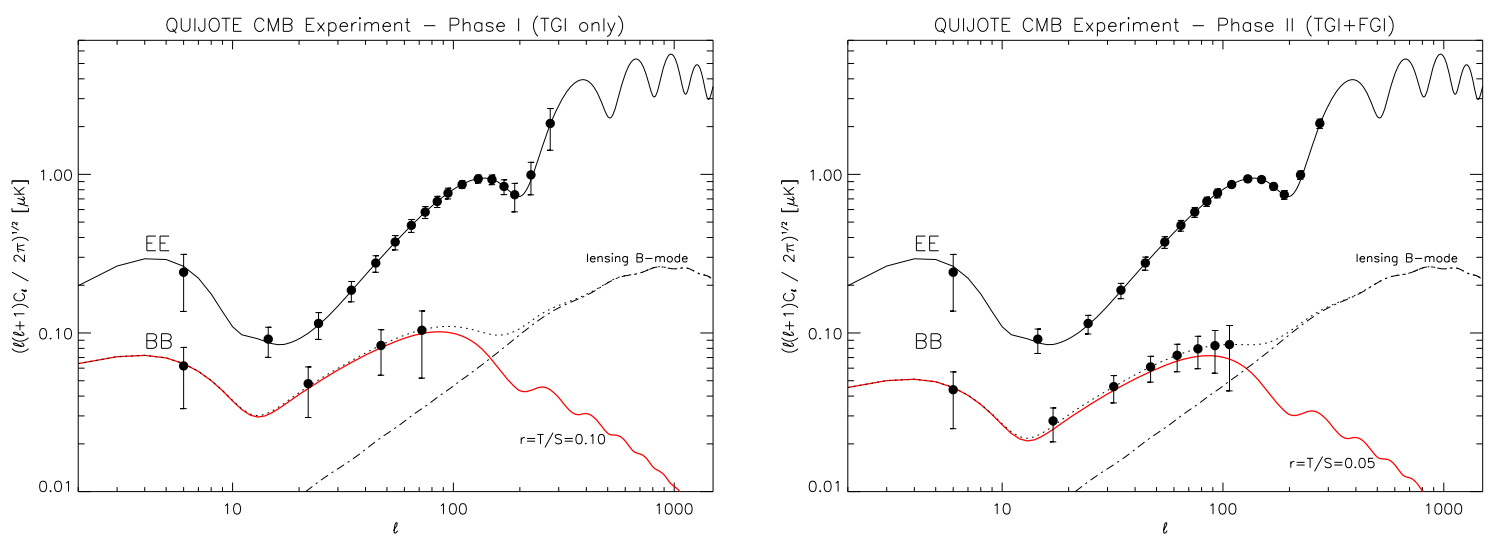

Figure 8. Left: Example of the QUIJOTE-CMB scientific goal after the Phase I of the project, for the angular power spectrum of the CMB E and B mode signals. It is shown the case for 1 year (effective) observing time, and a sky coverage of $\sim 3,000 \mathrm{deg}^{2}$. The red line corresponds to the primordial B-mode contribution in the case of $r=0.1$. Dots with error bars correspond to averaged measurements over a certain multipole band. Right: Same computation but now for the QUIJOTE-CMB Phase II. Here we consider 3 years of effective operations with the TGI, and that during the last 2 years, the FGI will be also operative. The red line now corresponds to $r=0.05$.

level for the $30 \mathrm{GHz}$ map is $\sim 0.5 \mu \mathrm{K} /$ beam. The right panel shows the scientific goal for the QUIJOTE-CMB Phase II. Here, we consider 3 years of effective observing time with the TGI, and 2 years with the FGI. Note that, once the two instruments (FGI and TGI) are available, they can be operated simultaneously, as we will have two telescopes. Finally, we stress that the computations presented in Fig. 8 correspond to the optimal situation in which the foreground removal leaves a negligible impact on the power spectrum. More realistic estimates will be published in a future paper.

\subsection{Non-core science}

Apart from the scientific goals described in the previous section, we have identified a number of secondary science projects. The characteristics of QUIJOTE-CMB make it a suitable experiment for performing (relatively-short) observations in specific regions that would allow us to tackle scientific objectives different to those for which it was conceived. Some of these possible projects are:

i) Study of the polarisation of Galactic regions and extragalactic sources. One of the main science drivers of $Q U I J O T E-C M B$ is to characterize the polarisation of the large-scale synchrotron emission from our Galaxy. 
However, it is also interesting to study this polarisation in specific Galactic regions, and also in extragalactic regions like M31, or in some of the 22 polarized sources detected in WMAP data. ${ }^{38}$ This could be done either with the MFI or with the polarized source subtractor, depending on the angular resolution.

ii) Study of the North Polar Spur. This a huge feature, visible mainly in radio wavelengths, which covers about a quarter of the sky and extends to high Galactic latitudes. Two main hypotheses have been proposed for its origin, namely a superbubble inflated by stellar winds and supernovae activity from the Scorpius-Centaurus OB association, on one hand, and an interaction between the loop I superbubble and the local superbubble. ${ }^{39}$ QUIJOTE-CMB data in this region may help to disentangle these two hypotheses.

iii) Study of the polarisation of the anomalous microwave emission (AME) in the Perseus molecular cloud and in other bright Galactic clouds. Apart from the synchrotron, it is also mandatory to have a good characterization of the AME polarisation in order to assess what level of contamination current and future Bmodes experiments will suffer. At present, only upper limits of the polarisation percentage have been obtained; ${ }^{40}$ these stand at $\sim 1 \%$ at the $95 \%$ C.L. We estimate that $35 \mathrm{~h}$ of observations with the $30 \mathrm{GHz}$ channels of the MFI on Perseus could allow us to obtain a $\sim 1 \%$ upper limit at the $99 \%$ C.L. Other possible targets include the $\rho$-Ophiuchi molecular cloud, ${ }^{41}$ the dark nebula LDN1622 or the Pleiades reflection nebula. ${ }^{42}$ These QUIJOTE$C M B$ measurements will provide a unique tool to understand the physical mechanism responsible for the AME, helping to distinguish between the electric dipole and the magnetic dipole radiation models. ${ }^{43,44}$

iv) Study of the WMAP haze in polarisation. This is an excess of microwave emission towards the centre of the Galaxy that was found at $23 \mathrm{GHz}$ in WMAP data, with a significantly flatter spectrum than synchrotron, and which has recently been shown to have a Gamma-ray counterpart in Fermi data. ${ }^{45}$ This is a burning subject at the moment, mainly owing to one of the proposed hypotheses for its origin, which is based on hard synchrotron radiation driven by relativistic electrons and positrons produced in the annihilations of one (or more) species of dark matter particles. ${ }^{46}$ QUIJOTE-CMB data could have an important contribution here, as it could allow us to measure, or to constrain the expected level of polarisation of this synchrotron emission.

v) Study of the polarisation of the WMAP cold spot. This is a non-Gaussian feature in the CMB, in the form of an extremely extended and cold region, which was found in WMAP data. ${ }^{47}$ After several considerations, it was proposed as a possible scenario for its origin the presence of a texture, a kind of topological defect which is predicted to occur in the primordial Universe. If this hypothesis were correct, a lack of polarisation would be expected in this region as compared with typical values of the primordial CMB. Therefore, the QUIJOTE-CMB data could help to disentangle the Gaussian and the texture hypotheses. In particular, it has been estimated ${ }^{48}$ that these data would be able to reject the Gaussian hypothesis with a significance of $~ 1 \%$.

\section{PROJECT STATUS AND TIMELINE}

QT1 is already installed at the Teide Observatory, and is now in the commissioning process. Immediately after this, the MFI will be commissioned, probably during this summer (2012). In parallel, the commissioning phase of the source-subtractor facility will take place.

We expect to install the QT2 at the end of 2013. Concerning the other two instruments, the TGI will be installed in the focal plane of the QT2, and will be commissioned by the end of 2013. The TGI will be available by the end of 2014. Note that once the TGI is operative, the FGI will be permanently installed in QT1, while the TGI will be placed in QT2.

\section{ACKNOWLEDGMENTS}

The QUIJOTE-CMB experiment is being developed by the Instituto de Astrofisica de Canarias (IAC), the Instituto de Fisica de Cantabria (IFCA), and the Universities of Cantabria, Manchester and Cambridge. Partial financial support is provided by the Spanish Ministry of Economy and Competitiveness (MINECO) under the projects AYA2010-21766-C03 (01, 02 and 03), and also by the Consolider-Ingenio project CSD2010-00064 (EPI: Exploring the Physics of Inflation $\left.{ }^{49}\right)$. 


\section{REFERENCES}

[1] Jarosik, N. et al., "Seven-year Wilkinson Microwave Anisotropy Probe (WMAP) Observations: Sky Maps, Systematic Errors, and Basic Results," ApJS 192, 14-+ (Feb. 2011).

[2] Dickinson, C. et al., "High-sensitivity measurements of the cosmic microwave background power spectrum with the extended Very Small Array," MNRAS 353, 732-746 (Sept. 2004).

[3] Reichardt, C. L. et al., "High-Resolution CMB Power Spectrum from the Complete ACBAR Data Set," ApJ 694, 1200-1219 (Apr. 2009).

[4] Readhead, A. C. S. et al., "Extended Mosaic Observations with the Cosmic Background Imager," ApJ 609, 498-512 (July 2004).

[5] Keisler, R. et al., "A Measurement of the Damping Tail of the Cosmic Microwave Background Power Spectrum with the South Pole Telescope," ApJ 743, 28 (Dec. 2011).

[6] Hlozek, R. et al., "The Atacama Cosmology Telescope: A Measurement of the Primordial Power Spectrum," ApJ 749, 90 (Apr. 2012).

[7] Komatsu, E. et al., "Seven-year Wilkinson Microwave Anisotropy Probe (WMAP) Observations: Cosmological Interpretation," ApJS 192, 18-+ (Feb. 2011).

[8] Planck Collaboration I, "Planck early results. I. The Planck mission," A $\& A$ 536, A1 (2011).

[9] Kovac, J. M., Leitch, E. M., Pryke, C., Carlstrom, J. E., Halverson, N. W., and Holzapfel, W. L., "Detection of polarization in the cosmic microwave background using DASI," Nature 420, 772-787 (Dec. 2002).

[10] Montroy, T. E. et al., "A Measurement of the CMB $<\mathrm{EE}>$ Spectrum from the 2003 Flight of BOOMERANG," ApJ 647, 813-822 (Aug. 2006).

[11] Sievers, J. L. et al., "Implications of the Cosmic Background Imager Polarization Data," ApJ 660, 976-987 (May 2007).

[12] Wu, J. H. P. et al., "MAXIPOL: Data Analysis and Results," ApJ 665, 55-66 (Aug. 2007).

[13] Bischoff, C. et al., "New Measurements of Fine-Scale CMB Polarization Power Spectra from CAPMAP at Both 40 and $90 \mathrm{GHz}, "$ ApJ 684, 771-789 (Sept. 2008).

[14] Brown, M. L. et al., "Improved Measurements of the Temperature and Polarization of the Cosmic Microwave Background from QUaD," ApJ 705, 978-999 (Nov. 2009).

[15] Chiang, H. C. et al., "Measurement of Cosmic Microwave Background Polarization Power Spectra from Two Years of BICEP Data," ApJ 711, 1123-1140 (Mar. 2010).

[16] Larson, D. et al., "Seven-year Wilkinson Microwave Anisotropy Probe (WMAP) Observations: Power Spectra and WMAP-derived Parameters," ApJS 192, 16 (Feb. 2011).

[17] QUIET Collaboration, Bischoff, C., et al., "First Season QUIET Observations: Measurements of Cosmic Microwave Background Polarization Power Spectra at $43 \mathrm{GHz}$ in the Multipole Range $25<\ell<475$," ApJ 741, 111 (Nov. 2011).

[18] Zaldarriaga, M. and Seljak, U., "All-sky analysis of polarization in the microwave background," Phys. Rev. D 55, 1830-1840 (Feb. 1997).

[19] Kamionkowski, M., Kosowsky, A., and Stebbins, A., "Statistics of cosmic microwave background polarization," Phys. Rev. D 55, 7368-7388 (June 1997).

[20] Polnarev, A. G., "Polarization and Anisotropy Induced in the Microwave Background by Cosmological Gravitational Waves," Soviet Astronomy 29, 607 (Dec. 1985).

[21] Partridge, B., "CMB observations and Cosmological Constraints," in [The Cosmic Microwave Background: from quantum fluctuations to the present Universe], Edited by J.A. Rubiño-Martin, R. Rebolo and E. Mediavilla CUP 2009, pp. 1-52 (2009).

[22] Bock, J. et al., "Task Force on Cosmic Microwave Background Research," ArXiv Astrophysics e-prints (Apr. 2006).

[23] Peacock, J. A. et al., "ESA-ESO Working Group on "Fundamental Cosmology"," tech. rep. (Oct. 2006).

[24] Rubiño-Martín, J. A. et al., "The QUIJOTE CMB Experiment," in [Highlights of Spanish Astrophysics V], Diego, J. M., Goicoechea, L. J., González-Serrano, J. I., and Gorgas, J., eds., 127 (2010).

[25] "QUIJOTE-CMB webpage." http://www.iac.es/project/cmb/quijote.

[26] "CMB webpage at the IAC." http://www.iac.es/project/cmb. 
[27] Gómez-Reñasco, F. et al., "Control system architecture of QUIJOTE multi-frequency instrument," Society of Photo-Optical Instrumentation Engineers (SPIE) Conference Series (2012).

[28] Gómez, A. et al., "QUIJOTE telescope design and fabrication," in [Society of Photo-Optical Instrumentation Engineers (SPIE) Conference Series], Society of Photo-Optical Instrumentation Engineers (SPIE) Conference Series $\mathbf{7 7 3 3}$ (July 2010).

[29] Hoyland, R. et al., "The status of the QUIJOTE I Multi-Frequency Instrument," Society of Photo-Optical Instrumentation Engineers (SPIE) Conference Series (2012).

[30] Cano, J. et al., "Multi-Pixel Ka-Band Radiometer for the QUIJOTE Experiment (Phase II)," Proc. of the 42nd European Microwave Conference (EuMC 2012), Amsterdam, The Netherlands (2012).

[31] Watson, R. A. et al., "First results from the Very Small Array - I. Observational methods," MNRAS 341, 1057-1065 (June 2003).

[32] Tucci, M., Martínez-González, E., Toffolatti, L., González-Nuevo, J., and De Zotti, G., "Predictions on the high-frequency polarization properties of extragalactic radio sources and implications for polarization measurements of the cosmic microwave background," MNRAS 349, 1267-1277 (Apr. 2004).

[33] Tucci, M., Martínez-González, E., Vielva, P., and Delabrouille, J., "Limits on the detectability of the CMB B-mode polarization imposed by foregrounds," MNRAS 360, 935-949 (July 2005).

[34] Kogut, A., "Synchrotron Spectral Curvature from $22 \mathrm{MHz}$ to $23 \mathrm{GHz}$, , ArXiv e-prints (May 2012).

[35] Ruiz-Granados, B., Rubiño-Martín, J. A., and Battaner, E., "Constraining the regular Galactic magnetic field with the 5-year WMAP polarization measurements at $22 \mathrm{GHz}, " A \mathscr{E} A$ 522, A73 (Nov. 2010).

[36] Watson, R. A., Rebolo, R., Rubiño-Martín, J. A., Hildebrandt, S., Gutiérrez, C. M., Fernández-Cerezo, S., Hoyland, R. J., and Battistelli, E. S., "Detection of Anomalous Microwave Emission in the Perseus Molecular Cloud with the COSMOSOMAS Experiment," ApJL 624, L89-L92 (May 2005).

[37] Battistelli, E. S., Rebolo, R., Rubiño-Martín, J. A., Hildebrandt, S. R., Watson, R. A., Gutiérrez, C., and Hoyland, R. J., "Polarization Observations of the Anomalous Microwave Emission in the Perseus Molecular Complex with the COSMOSOMAS Experiment," ApJL 645, L141-L144 (July 2006).

[38] López-Caniego, M., Massardi, M., González-Nuevo, J., Lanz, L., Herranz, D., De Zotti, G., Sanz, J. L., and Argüeso, F., "Polarization of the WMAP Point Sources," ApJ 705, 868-876 (Nov. 2009).

[39] Wolleben, M., Landecker, T. L., Reich, W., and Wielebinski, R., "An absolutely calibrated survey of polarized emission from the northern sky at $1.4 \mathrm{GHz}$. Observations and data reduction," $A \& A 448,411-$ 424 (Mar. 2006).

[40] López-Caraballo, C. H., Rubiño-Martín, J. A., Rebolo, R., and Génova-Santos, R., "Constraints on the Polarization of the Anomalous Microwave Emission in the Perseus Molecular Complex from Seven-year WMAP Data," ApJ 729, 25 (Mar. 2011).

[41] Dickinson, C., Peel, M., and Vidal, M., "New constraints on the polarization of anomalous microwave emission in nearby molecular clouds," MNRAS 418, L35-L39 (Nov. 2011).

[42] Génova-Santos, R., Rebolo, R., Rubiño-Martín, J. A., López-Caraballo, C. H., and Hildebrandt, S. R., "Detection of Anomalous Microwave Emission in the Pleiades Reflection Nebula with Wilkinson Microwave Anisotropy Probe and the COSMOSOMAS Experiment," ApJ 743, 67 (Dec. 2011).

[43] Draine, B. T. and Hensley, B., "Magnetic Nanoparticles in the Interstella Medium: Emission Spectrum and Polarization," ArXiv e-prints (May 2012).

[44] Draine, B. T. and Hensley, B., "The Submm and mm Excess of the SMC: Magnetic Dipole Emission from Magnetic Nanoparticles?," ArXiv e-prints (May 2012).

[45] Dobler, G., "A Last Look at the Microwave Haze/Bubbles with WMAP," ApJ 750, 17 (May 2012).

[46] Hooper, D., Finkbeiner, D. P., and Dobler, G., "Possible evidence for dark matter annihilations from the excess microwave emission around the center of the Galaxy seen by the Wilkinson Microwave Anisotropy Probe," Phys. Rev. D 76, 083012 (Oct. 2007).

[47] Vielva, P., Martínez-González, E., Barreiro, R. B., Sanz, J. L., and Cayón, L., "Detection of Non-Gaussianity in the Wilkinson Microwave Anisotropy Probe First-Year Data Using Spherical Wavelets," ApJ 609, 22-34 (July 2004).

[48] Vielva, P., Martínez-González, E., Cruz, M., Barreiro, R. B., and Tucci, M., "Cosmic microwave background polarization as a probe of the anomalous nature of the cold spot," MNRAS 410, 33-38 (Jan. 2011).

[49] "Consolider-Ingenio 2010 project: Exploring the Physics of Inflation." http://www.epi-consolider.es/. 